\title{
Secretin Measurement
}

National Cancer Institute

\section{Source}

National Cancer Institute. Secretin Measurement. NCI Thesaurus. Code C74871.

The determination of the amount of secretin hormone present in a sample. 